\title{
Patients with periodontitis and erectile dysfunction suffer a greater incidence of major adverse cardiovascular events: A prospective study in a Spanish population
}

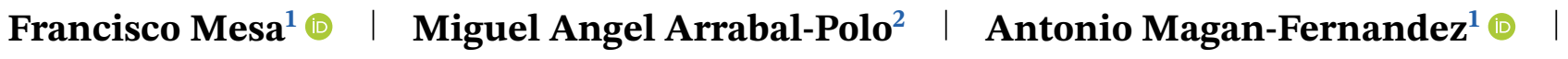 \\ Miguel Arrabal $^{2}$ | Amada Martin ${ }^{1}$ ｜ Ricardo Muñoz ${ }^{3}$ | Alejandro Rodriguez-Agurto ${ }^{1}$ \\ Manuel Bravo 4 (1)
}

${ }^{1}$ Department of Periodontics, School of Dentistry, University of Granada,

Granada, Spain

${ }^{2}$ Urology Unit, Granada University Hospital Complex, Granada, Spain

${ }^{3}$ Loja Healthcare Center, UGC Bucodental Metropolitano-Granada, Andalusian Public Health System, Granada, Spain

${ }^{4}$ Department of Preventive Dentistry and Epidemiology, School of Dentistry, University of Granada, Granada, Spain

\section{Correspondence}

Antonio Magán-Fernández, Facultad de Odontología, Campus de Cartuja s/n, Universidad de Granada, Granada 18071, Spain.

Email:amaganf@ugr.es

\begin{abstract}
Background: Periodontitis and erectile dysfunction (ED) have been linked with cardiovascular disease. The association of periodontitis and ED with the occurrence of major adverse cardiovascular events has not been previously assessed. The aim of this study was to determine if the presence of periodontitis and ED has any effect on the incidence of major adverse cardiovascular events.

Methods: Male patients that attended the Urology service were enrolled in a prospective study. Erectile dysfunction was diagnosed according to the International Index of Erectile Function. Sociodemographic data and periodontal clinical parameters were gathered (pocket probing depth, clinical attachment loss, bleeding on probing (BoP), plaque index and number of teeth) at baseline. Major adverse cardiovascular events occurred both before and during the follow-up time were registered. Bivariate analyses, as well as a multivariate analysis were performed, adjusting for potential confounders.

Results: A total of 158 patients were included, with a mean follow-up of 4.2 years. A greater number of major adverse cardiovascular events occurred in the group that presented periodontitis and $\operatorname{ED}(P=0.038)$. After adjusting by age and previous cardiovascular disease in the multivariate analysis, the annual major adverse cardiovascular event rate was estimated to be 3.7 times higher in the same group $(P=0.049)$. Other periodontal clinical variables together with ED supported these results and were close to statistical significance.

Conclusions: Patients with periodontitis and ED, adjusted by age and a cardiovascular disease, showed 3.7 times more risk of suffering major adverse cardiovascular events after mean follow-up of 4.2 years.
\end{abstract}




\section{KEYWORDS}

cardiovascular diseases, erectile dysfunction, longitudinal studies, periodontitis

\section{1 | INTRODUCTION}

Atherosclerotic cardiovascular disease is one of the most studied links between systemic disease and periodontitis, after type 2 diabetes mellitus. ${ }^{1}$ As shown in recent evidence, ${ }^{2,3}$ the atherosclerotic lesion begins with an endothelial dysfunction that can be early diagnosed through a decrease in the flow-mediated dilation (FMD) of the brachial artery. This ultrasonography assessment is considered the gold standard in vascular epidemiology. This evidence was confirmed by a meta-analysis in which an intima-media thickness $0.08 \mathrm{~mm}$ greater and a FMD 5.1\% lower was found in the internal carotid artery of patients with periodontitis. A mean improvement FMD of $6.6 \%$ was also found after successful periodontal therapy. ${ }^{4}$

Erectile dysfunction (ED) is a multifactorial pathology described as the repeated or continuous inability to achieve and maintain a sufficient penile erection to complete a satisfactory sexual intercourse, with a general prevalence in the male population of $24 \% .^{5}$ About $65 \%$ of ED cases are caused by an organic pathology, and within this, the inability of the vessels of the corpora cavernosa of the penis to accumulate blood and achieve an erection, is the most common cause. ${ }^{6}$ Based on the above evidence, it is reasonable to believe that periodontitis may be associated with ED through an atherosclerotic process in the vessels of the penis. The association between periodontitis and ED has been summarized by six systematic reviews, ${ }^{7-12}$ four of them with meta-analysis, ${ }^{7,8,10,11}$ and four observational studies with ORs between 1.29 and $2.75^{13-16}$ in the last 6 years.

Major Adverse Cardiovascular Events (MACEs) is an acronym used to describe a composite of cardiovascular clinical outcomes that address aspects of "safety" and "effectiveness." 17 Nonfatal ischemic stroke, nonfatal myocardial infarction, and cardiovascular death are commonly endpoints used as MACEs, but a standard definition has not yet been defined. ${ }^{18}$ The definition varies between studies, referring to a bigger composite endpoint, including heart failure, acute coronary syndrome (stable and unstable), coronary artery bypass grafting, or percutaneous coronary intervention. ${ }^{18}$ MACEs have been extensively used as a cardiovascular outcome after particular medical management or after undergoing certain procedures. ${ }^{19}$ Also, recent evidence has shown that periodontal disease inflammation is an independent predictor of the risk of MACEs, sug- gesting that arterial inflammation is the link behind this association. $^{20}$

Our study hypothesis (alternative) is that, given the smaller size of the penile vessels, the diagnosis of ED and periodontitis can be predictive and additive factors for future MACEs. The association of periodontitis and ED with the occurrence of MACEs has not been previously assessed in the literature. Our objective was to prospectively determine the incidence of MACEs in a group of patients with ED and periodontitis, and assess the joint risk of periodontitis and ED in the occurrence of MACEs.

\section{2 | MATERIALS AND METHODS}

A longitudinal observational study of two parallel cohorts was conducted in the Urology service of the "San Cecilio PTS” University Hospital (Granada, Spain). Cases were patients with ED, and controls were patients that attended the same service for different reasons and ED diagnosis was excluded, such as phimosis, vasectomy, lithotripsy, renal calculi, benign prostatic hyperplasia or family planning. Preliminary results from this group of patients were presented in a study published in $2018 .{ }^{16}$ The procedures in this study were in accordance with the Declaration of Helsinki, accordingly to the last revision in 2013. All participants signed written informed consent and before being enrolled in the study. The study protocol received approval from the Research Ethics Committee University Hospital (Ref. C-10.12). Strengthening the Reporting of Observational Studies in Epidemiology (STROBE) guidelines were followed for reporting the results of this manuscript. ${ }^{21}$

Inclusion criteria were the same as previously described in the previously published retrospective study. ${ }^{16}$ All the patients were of age between 18 and 70 years and they were required to have at least 11 present teeth. Participants were excluded if they had received previous periodontal treatment, if they were in treatment with atenolol, hydrochlorothiazide or had untreated hypertension (to exclude potential ED as side effect), if they had received antibiotic or anti-inflammatory therapy in the 2 months prior to the examination, if they presented any diagnosed psychiatric disorder and if they presented neoplastic diseases, HIV or other severe systemic infections. Sample size calculation was performed, and at least 126 subjects were necessary to detect a medium-sized standardized difference of 0.5 according to Cohen's ${ }^{22}$ and Sawilowsky's ${ }^{23}$ 
scales with a power of $80 \%$ and an alpha error of $5 \% .{ }^{16}$ The final sample size was of 158 patients, which provided an increased statistical power of $88 \%$. All sample size calculations and power analyses were performed using a specific software*.

ED was diagnosed by two urologists (M.A. and M-A.A.) at the time of consultation, following the International Index of Erectile Function, ${ }^{24}$ along with sociodemographic data for all participants on age, consumption of alcohol (grams per day) and tobacco (cigarettes per day), as well as comorbidities such as previous cardiovascular disease or diabetes. Periodontal clinical variables were determined by clinical examination by a previously calibrated dentist (A.M.). The calibration procedure was performed using an experienced researcher as reference (F.M.). Intraclass correlation coefficients were calculated for probing pocket depth (PPD), accepting $\pm 1 \mathrm{~mm}$ variability between measurements, and obtaining a result of $0.79 .{ }^{16}$ The researcher who performed all clinical examinations was blinded to the cohort allocation of each patient after urologic consultation, and offered to receive periodontal care at the school of Dentistry if periodontitis was diagnosed during the examination. The number of present teeth, PPD, gingival recession, clinical attachment loss, bleeding on probing $(\mathrm{BoP})^{25}$ and plaque index ${ }^{26}$ were registered. Periodontitis case definition was performed according to the criteria by López et al., ${ }^{27}$ which would be equivalent to a Stage II or higher of periodontitis according to the new classification of the European Federation of Periodontology (EFP) and American Academy of Periodontology (AAP) of $2018 .{ }^{28}$ Periodontitis severity was assessed through the Periodontal Inflammatory Severity Index (PISI) score, as the sum of the product of the number of sites and the PPD at each site divided by the number of remaining teeth (PISI $=\Sigma($ di ni $) / t$, where " $i$ " is the site, " $d$ " is the PPD of the site in mm, " $n$ " is the absolute frequency of the sites, and $t$ is the number of remaining teeth. ${ }^{16}$ For periodontitis extension, the criteria defined by Arbes et al., considering the percentage of sites per subject with clinical attachment loss $\geq 3 \mathrm{~mm} .{ }^{29}$

The follow-up went from January 2015 to March 2020. A dentist (A.R.) reviewed the hospital medical records of each patient to obtain data about newly diagnosed comorbidities and the occurrence of MACEs. Also, telephone calls were performed to each patient in order to obtain confirmation and further information if available, regarding MACEs in all the study participants. The information was collected in a specific confidential spreadsheet for this purpose. The MACEs included in the study were: nonfatal ischemic stroke, non-fatal myocardial infarction, cardiovascular death, heart failure, acute coronary syndrome

\footnotetext{
${ }^{*}$ Sample Power 2.0 (IBM Inc., Chicago, IL).
}

(stable and non-stable angina), coronary artery bypass graft or percutaneous coronary intervention.

\section{$2.1 \quad$ Statistical analysis}

To analyze the impact of having simultaneously both conditions, ED and periodontitis, on the risk of MACEs events compared with any other health situation, having none or just one of these two conditions, was considered. This consideration was also tested with the rest of the periodontal clinical variables assessed in the study. The cut-off point used for the corresponding continuous variables was the median. For variables of interest an exploratory analysis was carried out to describe their univariate and bivariate characteristics, considering ANOVA in case of a continuous normally distributed variable, and a Kruskal-Wallis test in case of a non-normal continuous variable. In case of two binary variables the Fisher's exact test was applied.

MACEs were assumed to follow a Poisson-distribution and modelled using a General Linear Model (GLM) with logarithmic link function. ${ }^{30}$ To adjust for different length of observations an offset with respect to the follow-up time was included into the model. As a consequence, the risk of MACEs is expressed as an occurrence-rate relative to the time-unit year. Among the adjusting variables considered were age, smoking habits, drinking habit, the presence of diabetes and the presence of a previously diagnosed cardiovascular condition. Variables were included into the model according to their significance level as well as to the Akaike Information Criterion; also, bivariate interactions terms were considered. To ensure absence of any substantial overdispersion of the estimated GLM a dispersion-test suggested by Cameron and Trivedi was applied. ${ }^{31,32}$ For the variable of interest marginal means of the rates were estimated together with their $95 \%$-confidence intervals. ${ }^{33}$ Furthermore, contrasts were calculated to reflect the ratio of estimates of the two population-groups together with the corresponding $P$-values. All analyses were performed using a specific statistical software package ${ }^{\dagger}$. Statistical significance threshold was $P \leq 0.05$. Statistical analyses are detailed in table footnotes.

\section{3 | RESULTS}

Data from 158 patients with information on ED and periodontitis were included in the analysis with a mean follow-up time was $4.2 \pm 0.9$ years. No patient diagnosed with periodontitis reported to receive periodontal care during the follow-up period. In three patients, only

${ }^{\dagger}$ RStudio in R-4.0.2 (RStudio, PBC, Boston, MA). 
partial follow-up data was gathered, due two of them that had incomplete medical records, had moved away and were not traceable by phone, and other one that died of cancer. However, these patients provided 38.9, 36.4, and 49.1 months of follow-up for the study, respectively. Sociodemographic variables related to age, previous diagnosis of diabetes and cardiovascular diseases (CVD), smoking and drinking habit, were already described in the original case-control study. ${ }^{16}$ Baseline description of sociodemographic variables, including age, smoking habit, drinking habit, diabetes and CVD, and periodontal clinical variables associated with ED in the patients of the study are detailed in Table 1 .

In Table 2, the number of MACEs registered (0, 1, and 2) before the study start are described and compared with the sociodemographic and each periodontal variable together with ED. The only variable significantly associated with a higher occurrence of MACEs before the study, was a previous diagnosis of CVD, as expected. Table 3 describes the occurrence of MACEs until the study ended, following the same structure. In the group of patients with ED and Periodontitis, seven of them suffered a total of 10 MACEs (three patients suffered two events), compared to the rest of the patients, in which only three patients suffered one event each $(P=0.038)$. Diagnosis of ED together with high values of BoP and PISI, showed a relationship with higher number of MACEs during the study close to statistical significance. Previous diagnosis of CVD was also associated with higher number of MACEs during the study.

Table 4 depicts the comparison between each sociodemographic variable with each periodontal clinical variable together with ED. No statistical differences were found regarding any of these variables in the group of patients with both ED and periodontitis compared to the other groups. Patients with ED and higher levels of BoP and plaque index were associated with diabetes and CVD.

Table 5 describes the results of the GLM following the Poisson distribution, where the MACEs occurrence and the presence of ED and periodontitis is assessed, adjusting for age and CVD. After the adjustment, the higher occurrence of MACEs in the ED and periodontitis group was still statistically significant $(P=0.049)$. Means and contrasts were calculated in Table 6 to provide a ratio of the occurrence of MACEs between the two comparison groups, showing that the ED and periodontitis group presented a rate of $0.018 \mathrm{MACEs} / \mathrm{y}$ compared to 0.005 MACEs/y in the rest of the groups. This difference led to a risk 3.7 higher of developing MACEs. The same model was applied to the rest of periodontal variables together with ED, and although PISI and IG supported the tendency of results obtained with periodontitis, statistical significance was close to but did not achieve significance $(P=0.064$ and $P=0.094$, respectively).
TA B LE 1 Descriptive baseline sociodemographic and erectile dysfunction-periodontal variables in the study patients $(n=158)$

\begin{tabular}{|c|c|}
\hline Variable & Value \\
\hline Age, mean (SD) & $52.8(8.7)$ \\
\hline \multicolumn{2}{|l|}{ Smoker } \\
\hline No $(\%)$ & $122(77.2)$ \\
\hline Yes $(\%)$ & $36(22.8)$ \\
\hline Tobacco (Cigs. /d), mean (SD) & $3.0(7.1)$ \\
\hline \multicolumn{2}{|l|}{ Drinker } \\
\hline No (\%) & $57(36.1)$ \\
\hline Yes $(\%)$ & $101(63.9)$ \\
\hline Alcohol (g/d), mean (SD) & $9.2(12.7)$ \\
\hline \multicolumn{2}{|l|}{ Diabetes } \\
\hline No $(\%)$ & $131(82.9)$ \\
\hline Yes $(\%)$ & $27(17.1)$ \\
\hline \multicolumn{2}{|l|}{ Cardiovascular disease } \\
\hline No (\%) & $87(55.1)$ \\
\hline Yes $(\%)$ & $71(44.9)$ \\
\hline \multicolumn{2}{|l|}{ ED_Periodontitis } \\
\hline Yes $(\%)$ & $59(37.3)$ \\
\hline Other (\%) & 99 (62.7) \\
\hline \multicolumn{2}{|l|}{ ED_PISI ${ }^{\mathrm{a}}$} \\
\hline Yes $(\%)$ & $44(27.8)$ \\
\hline Other $(\%)$ & $114(72.2)$ \\
\hline \multicolumn{2}{|l|}{ ED_ARBES ${ }^{\mathrm{a}}$} \\
\hline Yes $(\%)$ & 47 (29.7) \\
\hline Other (\%) & $111(70.3)$ \\
\hline \multicolumn{2}{|l|}{ ED_BoP } \\
\hline Yes $(\%)$ & $44(27.8)$ \\
\hline Other (\%) & $114(72.2)$ \\
\hline \multicolumn{2}{|l|}{ ED_Plaque index } \\
\hline Yes (\%) & $40(25.3)$ \\
\hline Other $(\%)$ & $118(74.7)$ \\
\hline
\end{tabular}

Abbreviations: ED, Erectile dysfunction; BoP, Bleeding on Probing. a PISI (variable related to periodontal severity) and ARBES (variable related to periodontal extension) described in Martin et al., 2018. ${ }^{16}$

Each ED-periodontal parameter variable was constructed accounting for the patients that presented both conditions. In the case of continuous variables, such as PISI or ARBES, the chosen cut-off points for considering them as affirmative was the median.

\section{4 | DISCUSSION}

The main question considered here was whether patients having simultaneously both conditions ED and periodontitis face a higher risk of experiencing MACEs than those patients having just one of the conditions or none. In patients with both pathologies, periodontitis presence (given its pathogenesis, chronicity and evolution) would be already present when the diagnosis of ED is performed in most cases. ED is a disease that, because of its 
TA B LE 2 Bivariate comparisons of MACEs before the beginning of the study

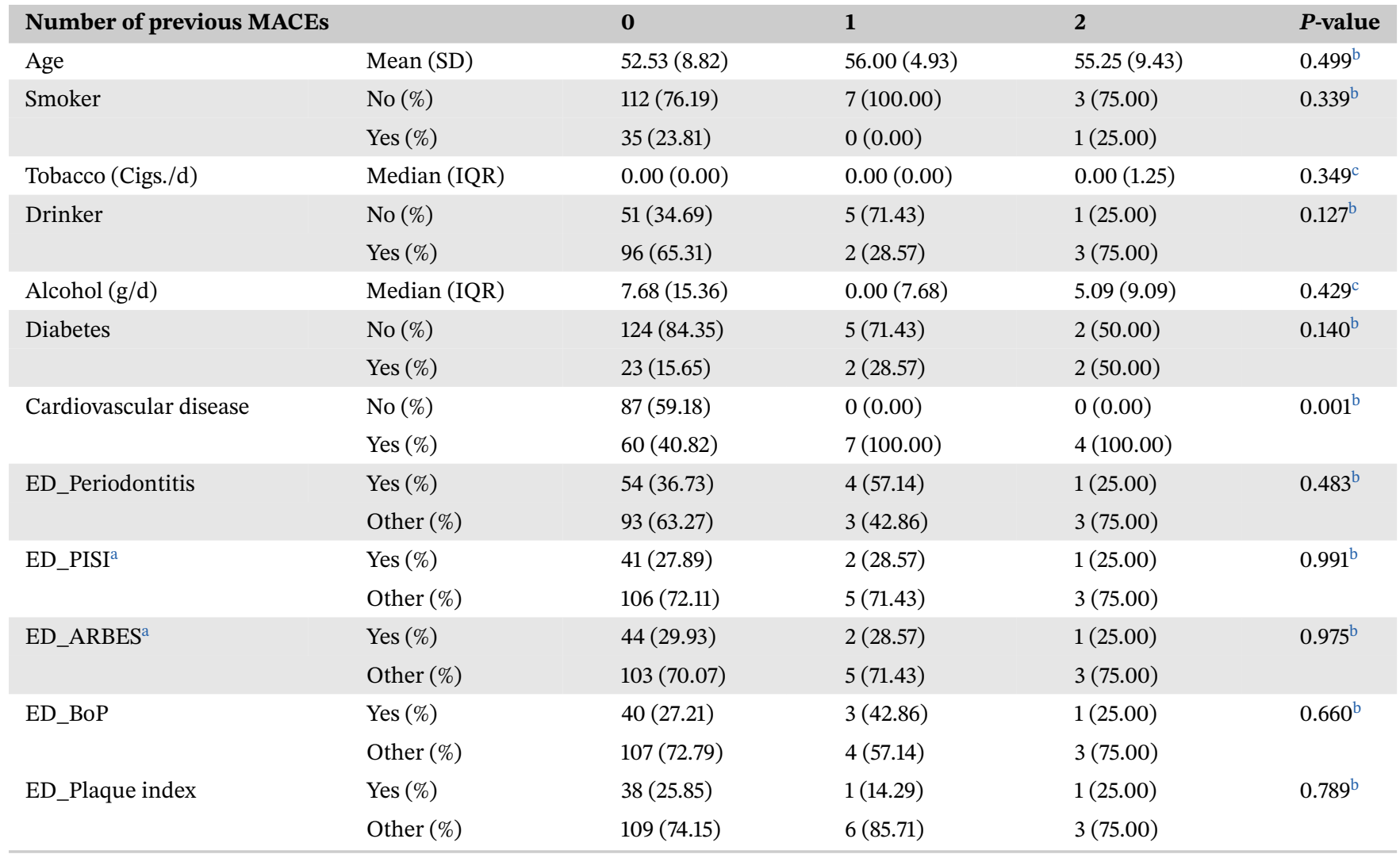

Abbreviations: ED, Erectile dysfunction; BoP, Bleeding on Probing; MACEs, Major Adverse Cardiovascular Events.

Each ED-periodontal parameter variable was constructed accounting for the patients that presented both conditions. In the case of continuous variables, such as PISI or ARBES, the chosen cut-off points for considering them as affirmative was the median.

${ }^{\text {a }}$ PISI (variable related to periodontal severity) and ARBES (variable related to periodontal extension) described in Martin et al., 2018. ${ }^{16}$

${ }^{\mathrm{b}}$ ANOVA.

${ }^{\mathrm{c}}$ Kruskall-Wallis test.

personal implications, leads to a quicker medical consultation by the patient. Periodontitis, when usually diagnosed, has already been progressing for years and therefore could be a risk factor for ED and therefore, an early predictor of cardiovascular disease given their relationship with endothelial dysfunction.

To our knowledge, these are the first results to demonstrate the association with MACEs in patients with ED and periodontitis in a follow-up study which reached to 5.3 years in some patients. Its main finding was that patients with ED and periodontitis were associated with a higher occurrence of MACEs events compared to the other three groups (periodontitis without ED, patients without periodontitis with ED, patients without periodontitis and without ED). According to the results of this study, out of every 100 patients with periodontitis and ED, 1.8 people/y would suffer an event, this occurrence rate being 3.7 times higher than in an individual without these pathologies. The importance of being able to prevent MACEs is a first-order problem in public health, given that they are events with high morbidity and mortality, which constitute both a vital risk and a high possibility of leaving sequelae for life, in patients who suffer from them. ${ }^{34}$

The vessels of the corpora cavernosa of the penis are smaller than the coronary arteries; patients with an atherosclerotic pathology are more likely to initially present with ED and then develop a cardio-cerebrovascular lesion, with more serious clinical repercussions. Vlachopoulos et al., reported that the prevalence of ED was $47 \%$ in patients with cardiovascular events, a figure much higher than the $24 \%$ prevalence of ED in the general population. In addition, they demonstrated that the severity (IIEF < 10) and the duration (>24 months) of the ED indicated a greater coronary compromise measured by angiography. ${ }^{35}$ These data support the hypothesis of considering ED as an early diagnosis manifestation to predict a future MACEs. Two recent studies, one of them a metaanalysis, concluded that ED is a predictor of future cardiovascular events, independently of other associated risk factors. ${ }^{36,37}$

In this study, performed in patients with ED and subsequently diagnosed with periodontitis, we confirmed 
TA B L E 3 Bivariate comparisons of MACE events after the study start

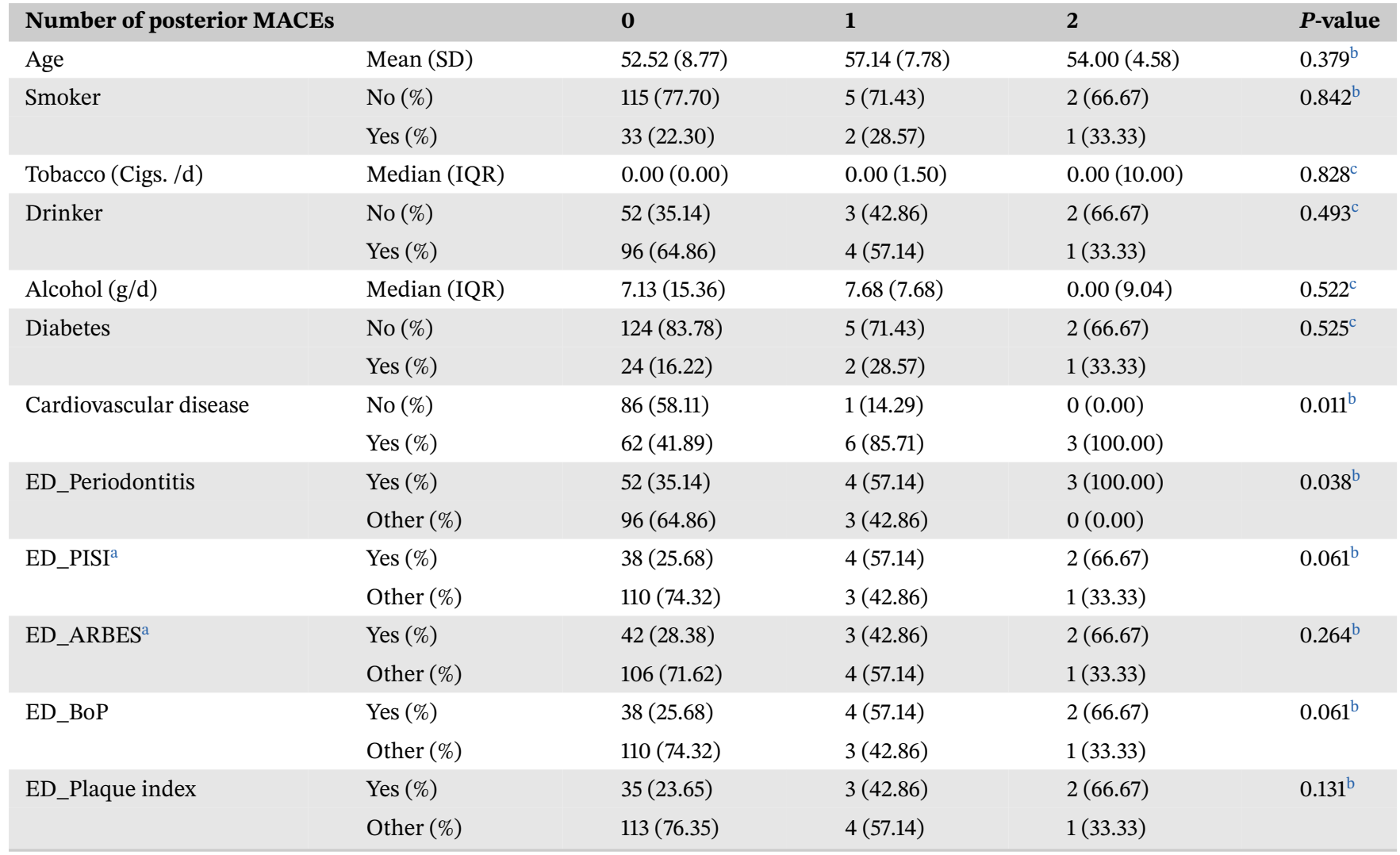

Abbreviations: ED, Erectile dysfunction; BoP, Bleeding on Probing; MACEs, Major Adverse Cardiovascular Events.

Each ED-periodontal parameter variable was constructed accounting for the patients that presented both conditions. In the case of continuous variables, such as PISI or ARBES, the chosen cut-off points for considering them as affirmative was the median.

${ }^{a}$ PISI (variable related to periodontal severity) and ARBES (variable related to periodontal extension) described in Mart in et al., 2018. ${ }^{16}$

bANOVA.

${ }^{\mathrm{c}}$ Kruskall-Wallis test.

and quantified the highest occurrence of MACEs. Seven patients with both diagnoses, suffered 10 MACEs events (three patients, two events), compared to three patients with a single event each, in the other groups (no patient with two events). Although these differences are statistically significant (adjusted $P$-value $=0.049$ ), we think that these results would have been even higher if the periodontitis evolution time had been longer (mean age of 52.52 years in the ED_Periodontitis group). Similarly, the periodontal clinical variables PISI and BoP with ED were close to statistical significance when related to the occurrence of MACEs. Chou et al., reported results in Taiwanese adult population, treated for periodontitis, with a slight but significant risk association between treated severe periodontitis and MACEs in patients older than 60 years and not in patients younger than this age, ${ }^{38}$ these authors, however, they included two events, malignant dysrhythmia and thrombolysis, which we did not include in our study. Other studies have recently described that the heterogeneity often found in the periodontitis and systemic diseases association might be explained by a combination of genetic, environmental and behavioral factors that constitute a biological age, that may be different to the most used chronological one in each patient. ${ }^{39}$

The relationship of patients with a single pathology (periodontitis or ED) and the occurrence of serious cardiovascular events and/or MACEs have been recently studied. A prospective 3-year study performed on German population did not find any statistical significance when analyzing the occurrence of a composite endpoint of several CVD similar to MACEs in patients with periodontitis, although patients with severe periodontitis achieved this combined CVD endpoint more often $(\mathrm{HR}=1.05)$ and other periodontal parameters where statistically significant such as BoP and missing teeth. These results may be explained by a short follow-up period compared to our study and other published evidence, despite the significantly big sample size of 1000 patients approximately. ${ }^{40}$ Another study performed in a cohort of patients selected from the Swedish National Study of Aging and Care, a prospective 17-year follow-up study, Periodontitis increased the risk for all-cause mortality in men, with a hazard ratio of 


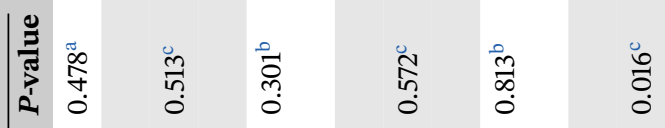

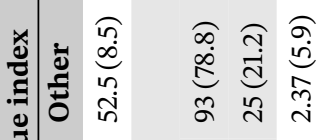

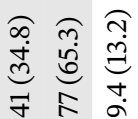

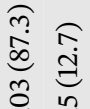

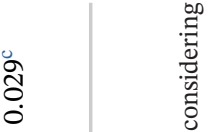

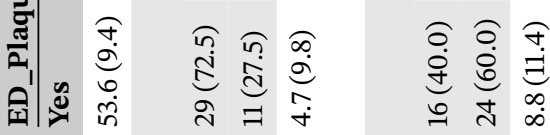

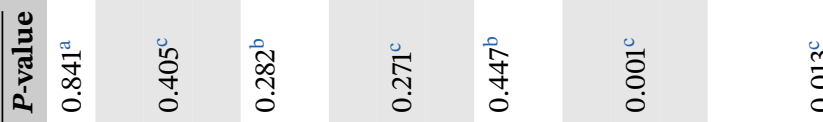

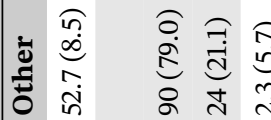

त̂?

䇥

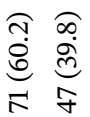

ôे

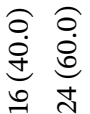

$\stackrel{m}{\circ}$

离

a $\approx$ ก

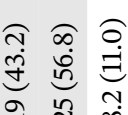

羟

贾

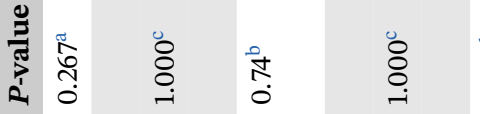

峁

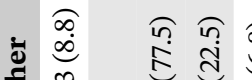

छ 的

ㄷํㅇ ป

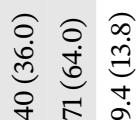

赵

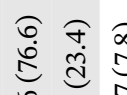

$\infty$ o

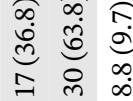

ㄱำ

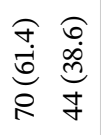

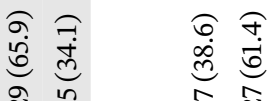

ते थ

o

苛

¿ ำ

家

告

焉

苞

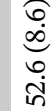

ำ

อิ

ஜ्ల

F

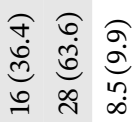

ֻٓ

$\stackrel{\check{\infty}}{\dddot{0}}$

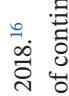

同产

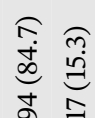

苟

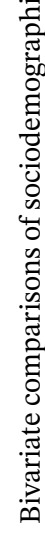

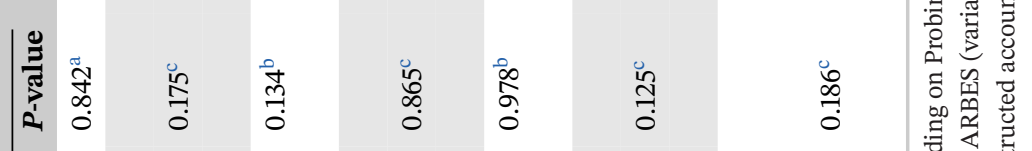

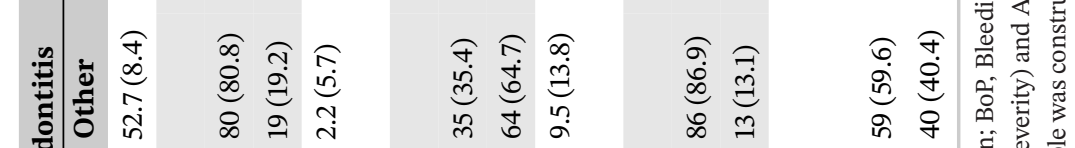

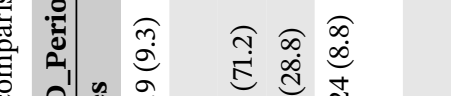

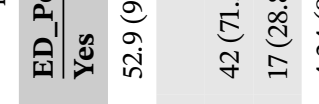

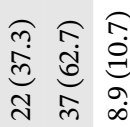

זิ

의

๙ิ

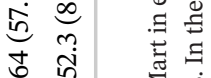


TA B LE 5 General linear model with Poisson regression of the relationship between MACEs and the variable ED_Periodontitis

\begin{tabular}{|lllll} 
Variable & Estimate & Std. error & $\boldsymbol{z}$ value & $\operatorname{Pr}(>|\mathbf{z}|)$ \\
\hline (Intercept) & -4.670 & 2.232 & -2.093 & 0.036 \\
$\begin{array}{l}\text { ED_Periodontitis } \\
\quad-1.307\end{array}$ & 0.664 & -1.968 & 0.049 \\
$\quad$ (Other) & & & & \\
Age & -0.013 & 0.041 & -0.307 & 0.759 \\
CVD (Yes) & 2.682 & 1.106 & 2.425 & 0.015 \\
\hline
\end{tabular}

ED: Erectile dysfunction; MACEs: Major Adverse Cardiovascular Events; CVD: Cardiovascular Disease.

ED_Periodontitis was constructed accounting for the patients that presented both conditions.

Model adjusted for the variables age and CVD.

1.5. ${ }^{41}$ Other study in Danish population reported a 2.02 rate cardiovascular death in patients with periodontitis after a 15 -year follow-up. ${ }^{42}$ Despite having different follow-up periods, these studies present lower rates than the one reported in our study. This could be explained by the fact that in our study both diseases together (ED and Periodontitis) are considered. Also, these studies use different outcomes (all-cause mortality and cardiovascular death, respectively) compared to the ones considered in our study (MACEs) that include a wider variety of events, and not only mortality-related ones.

Regarding the association of ED with MACEs, a study performed as a sub-sample of the Multi-Ethnic Study of Atherosclerosis, a prospective 7-year follow-up cohort study, ED was a significant predictor of CVD events, with a ratio of $1.9 .^{43}$ In a recent systematic review and meta-analysis, performed in 25 studies, risk of total CVD $(\mathrm{RR}=1.43)$, coronary heart disease $(\mathrm{RR}=1.59)$ and allcause mortality $(R R=1.33)$ were significantly increased in ED. ${ }^{37}$ Likewise, most of the studies involved in this review were carried out in a population older than 55 years (older than that of the present study) and most of the followup times were longer than 7 years, compared to 4.2 years of mean follow-up of the present study. In summary, taking into account the presence of both pathologies together, more powerful results are obtained in terms of association, they occur in a younger population, and in a shorter followup period.

For the case definition of periodontitis, criteria different from those currently recommended by the EFP in the new classification of periodontal and peri-implant diseases have been followed. We would like to highlight that this research project began in 2014, with a patient recruitment period until 2016. In this period, the new case definition of periodontitis from the EFP and the AAP was not still available. ${ }^{28}$ In addition, the criteria established by López et al, are more strict than those of the EFP/AAP, because they consider as a case, that patient with a PPD of $\geq 4 \mathrm{~mm}$ in $\geq 4$ different teeth, with clinical attachment loss of $\geq 3 \mathrm{~mm}$ and BoP, at these same sites, ${ }^{27}$ whereas the new case definition of periodontitis considers an interdental clinical attachment loss of $>3 \mathrm{~mm}$ in $\geq 2$ different teeth, or in buccal or lingual sites together with $\geq 4 \mathrm{~mm}$ PPD. ${ }^{28}$

Among this study strengths are being the first that jointly assesses these three diseases. The previously published case-control (retrospective study) ${ }^{16}$ and the present prospective one are both longitudinal studies of high evidence level. Also, the evidence about the role of ED and periodontitis is scarce in European population, because the majority of the studies have been performed on Asian population. ${ }^{13-15,44-47}$ As potential limitations of this study, the use of medical records as source for the assessed outcomes could have led to a loss of information. This information bias was managed by contacting each subject by phone as possible and ensure that the data in medical records were precise. Although the follow-up period of the present study is long, longer follow-up periods are needed in order to assess with greater accuracy the incidence of MACEs, because cardiovascular disease outcomes have a long latency. The borderline results of the $P$-values are also a potential limitation and should be viewed with caution, until further confirmation by posterior studies with longer follow-ups or multicentric designs. Also, another potential limitation is that the role of phosphodiesterase-5 inhibitors in the occurrence of MACEs has not been evaluated. Although they are commonly used drugs in these patients for improving erectile function, two reasons have influenced this decision. They are not included among the drugs covered and funded by the Spanish National Health System, and are only used on demand by patients who can afford them, and also making their registration and tracking of its usage in the medical records reliable enough.

TA B L E 6 Marginal means and contrast from general linear model: Incidence rate ratio of MACEs according to the variable ED_Periodontitis

\begin{tabular}{|llllllll} 
ED_Periodontitis & Rate & SE & 95\% CI & Ratio & SE & $\boldsymbol{z}$-ratio & $\boldsymbol{P}$-value \\
\hline Yes & 0.018 & 0.010 & $0.006-0.054$ & 3.693 & 2.452 & 1.968 & 0.049 \\
Other & 0.005 & 0.004 & $0.001-0.020$ & & & & \\
\hline
\end{tabular}

Abbreviations: ED, Erectile dysfunction; MACEs, Major Adverse Cardiovascular Events.

ED_Periodontitis was constructed accounting for the patients that presented both conditions. 


\section{5 | CONCLUSION}

In conclusion and within the limitations of the present study patients with periodontitis and ED, adjusted by age and CVD, showed 3.7 times more risk of suffering MACEs after a mean follow-up 4.2 years.

\section{ACKNOWLEDGMENTS}

This investigation was supported by Research Group \#CTS-583 (Junta de Andalucía, Granada, Spain).

\section{CONFLICTS OF INTEREST}

The authors report no conflicts of interest related to this study.

\section{DATA AVAILABILITY STATEMENT}

The data that support the findings of this study are available on request from the corresponding author. The data are not publicly available because of privacy or ethical restrictions.

\section{O R C I D}

Francisco Mesa (1) https://orcid.org/0000-0001-8943-9068 Antonio Magan-Fernandez (10 https://orcid.org/0000-

0001-6430-2276

Manuel Bravo (1D https://orcid.org/0000-0001-5508-561X

\section{REFERENCES}

1. Schenkein HA, Papapanou PN, Genco R, Sanz M. Mechanisms underlying the association between periodontitis and atherosclerotic disease. Periodontol 2000. 2020;83:90-106.

2. Moura MF, Navarro TP, Silva TA, Cota LOM, Soares Dutra Oliveira AM, Costa FO. Periodontitis and endothelial dysfunction: periodontal clinical parameters and levels of salivary markers interleukin-1beta, tumor necrosis factor-alpha, matrix metalloproteinase-2, tissue inhibitor of metalloproteinases-2 complex, and nitric oxide. J Periodontol. 2017;88:778-787.

3. Punj A, Shenoy SB, Subramanyam K. Comparison of endothelial function in healthy patients and patients with chronic periodontitis and myocardial infarction. J Periodontol. 2017;88:1234-1243.

4. Orlandi M, Suvan J, Petrie A, et al. Association between periodontal disease and its treatment, flow-mediated dilatation and carotid intima-media thickness: a systematic review and metaanalysis. Atherosclerosis. 2014;236:39-46.

5. Montorsi F, Adaikan G, Becher E, et al. Summary of the recommendations on sexual dysfunctions in men. J Sex Med. 2010;7:3572-3588.

6. Chiurlia E, D'Amico R, Ratti C, Granata AR, Romagnoli R, Modena MG. Subclinical coronary artery atherosclerosis in patients with erectile dysfunction. J Am Coll Cardiol. 2005;46:1503-1506.

7. Wang Q, Kang J, Cai X, Wu Y, Zhao L. The association between chronic periodontitis and vasculogenic erectile dysfunction: A systematic review and meta-analysis. J Clin Periodontol. 2016;43:206-215.
8. Liu LH, Li EM, Zhong SL, et al. Chronic periodontitis and the risk of erectile dysfunction: a systematic review and metaanalysis. Int J Impot Res. 2017;29:43-48.

9. Kellesarian SV, Kellesarian TV, Ros Malignaggi V, et al. Association between periodontal disease and erectile dysfunction: a systematic review. Am J Men's Health. 2018;12:338-346.

10. Zhou X, Cao F, Lin Z, Wu D. Updated evidence of association between periodontal disease and incident erectile dysfunction. $J$ Sex Med. 2019;16:61-69.

11. Farook F, Al Meshrafi A, Mohamed Nizam N, Al Shammari A. The association between periodontitis and erectile dysfunction: a systematic review and meta-analysis. Am J Men's Health. 2021;15:15579883211007277.

12. Lecaplain B, Badran Z, Soueidan A, Prud'homme T, Gaudin A. Periodontitis, erectile dysfunction, reproductive hormones, and semen quality: a systematic review. Andrology. 2021;9:769-780.

13. Lee JH, Lee JS, Park JY, et al. Association of lifestyle-related comorbidities with periodontitis: a nationwide cohort study in Korea. Medicine (Baltimore). 2015;94:e1567.

14. Tsao CW, Liu CY, Cha TL, Wu ST, Chen SC, Hsu CY. Exploration of the association between chronic periodontal disease and erectile dysfunction from a population-based view point. Andrologia. 2015;47:513-518.

15. Lee JH, Choi JK, Kim SH, et al. Association between periodontal flap surgery for periodontitis and vasculogenic erectile dysfunction in Koreans. J Periodontal Implant Sci. 2017;47:96-105.

16. Martin A, Bravo M, Arrabal M, Magan-Fernandez A, Mesa F. Chronic periodontitis is associated with erectile dysfunction. A case-control study in European population. J Clin Periodontol. 2018;45:791-798.

17. Heianza Y, Ma W, Manson JE, Rexrode KM, Qi L. Gut microbiota metabolites and risk of major adverse cardiovascular disease events and death: a systematic review and meta-analysis of prospective studies. J Am Heart Assoc. 2017;6:e04947.

18. Kip KE, Hollabaugh K, Marroquin OC, Williams DO. The problem with composite end points in cardiovascular studies: the story of major adverse cardiac events and percutaneous coronary intervention. J Am Coll Cardiol. 2008;51:701-707.

19. Shima D, Ii Y, Higa S, et al. Validation of novel identification algorithms for major adverse cardiovascular events in a Japanese claims database. J Clin Hypertens (Greenwich). 2021;23:646-655.

20. Van Dyke TE, Kholy KE, Ishai A, et al. Inflammation of the periodontium associates with risk of future cardiovascular events. $J$ Periodontol. 2021;92:348-358.

21. von Elm E, Altman DG, Egger M, et al. The Strengthening the Reporting of Observational Studies in Epidemiology (STROBE) statement: guidelines for reporting observational studies. J Clin Epidemiol. 2008;61:344-349.

22. Cohen J. Statistical Power Analysis for the Behavioural Sciences. Lawrence Erlbaum Associates; 1988.

23. Sawilowsky SS. New effect size rules of thumb. J Mod Appl Stat Methods. 2009;8:597-599.

24. Rosen RC, Cappelleri JC, Gendrano N 3rd. The International Index of Erectile Function (IIEF): a state-of-the-science review. Int J Impot Res. 2002;14:226-244.

25. Ainamo J, Bay I. Problems and proposals for recording gingivitis and plaque. Int Dent J. 1975;25:229-235. 
26. Tonetti MS. The future of periodontology: new treatments for a new era. J Int Acad Periodontol. 2002;4:110-114.

27. Lopez NJ, Smith PC, Gutierrez J. Periodontal therapy may reduce the risk of preterm low birth weight in women with periodontal disease: a randomized controlled trial. J Periodontol. 2002;73:911-924.

28. Tonetti MS, Greenwell H, Kornman KS. Staging and grading of periodontitis: framework and proposal of a new classification and case definition. J Clin Periodontol. 2018;45(Suppl 20):S149S161.

29. Arbes SJ Jr., Slade GD, Beck JD. Association between extent of periodontal attachment loss and self-reported history of heart attack: an analysis of NHANES III data. J Dent Res. 1999;78:17771782.

30. Fahrmeir L, Tutz G. Multivariate Statistical Modelling Based on Generalized Linear Models. Springer; 2001.

31. Cameron AC, Trivedi PK. Regression-based tests for overdispersion in the poisson model. J Econom. 1990;46:347-364.

32. Kleiber C, Zeileis A. Applied Econometrics with R. SpringerVerlag; 2008.

33. Searle SR, Speed FM, Milliken GA. Population marginal means in the linear model: an alternative to least squares means. $\mathrm{Am}$ Stat. 2012;34:216-221.

34. Cassar A, Holmes DR Jr., Rihal CS, Gersh BJ. Chronic coronary artery disease: diagnosis and management. Mayo Clin Proc. 2009;84:1130-1146.

35. Vlachopoulos CV, Terentes-Printzios DG, Ioakeimidis NK, Aznaouridis KA, Stefanadis CI. Prediction of cardiovascular events and all-cause mortality with erectile dysfunction: a systematic review and meta-analysis of cohort studies. Circ Cardiovasc Qual Outcomes. 2013;6:99-109.

36. Vlachopoulos C, Ioakeimidis N, Rokkas K, et al. Central haemodynamics and prediction of cardiovascular events in patients with erectile dysfunction. Am J Hypertens. 2017;30:249-255.

37. Zhao B, Hong Z, Wei Y, Yu D, Xu J, Zhang W. Erectile dysfunction predicts cardiovascular events as an independent risk factor: a systematic review and meta-analysis. J Sex Med. 2019;16:1005-1017.

38. Chou SH, Tung YC, Lin YS, et al. Major adverse cardiovascular events in treated periodontitis: a population-based follow-up study from Taiwan. PLoS One. 2015;10:e0130807.
39. Meisel P, Nauck M, Kocher T. Individual predisposition and the intricate interplay between systemic biomarkers and periodontal risk in a general population. J Periodontol. 2021;92:844853.

40. Reichert S, Schulz S, Benten AC, et al. Periodontal conditions and incidence of new cardiovascular events among patients with coronary vascular disease. J Clin Periodontol. 2016;43:918-925.

41. Bengtsson VW, Persson GR, Berglund JS, Renvert S. Periodontitis related to cardiovascular events and mortality: a long-time longitudinal study. Clin Oral Investig. 2021;25:4085-4095.

42. Hansen GM, Egeberg A, Holmstrup P, Hansen PR. Relation of periodontitis to risk of cardiovascular and all-cause mortality (from a Danish Nationwide Cohort Study). Am J Cardiol. 2016;118:489-493.

43. Uddin SMI, Mirbolouk M, Dardari Z, et al. Erectile dysfunction as an independent predictor of future cardiovascular events: the multi-ethnic study of atherosclerosis. Circulation. 2018;138: 540-542.

44. Matsumoto S, Matsuda M, Takekawa M, et al. Association of ED with chronic periodontal disease. Int J Impot Res. 2014;26:13-15.

45. Keller JJ, Chung SD, Lin HC. A nationwide population-based study on the association between chronic periodontitis and erectile dysfunction. J Clin Periodontol. 2012;39:507-512.

46. Zuo Z, Jiang J, Jiang R, et al. Effect of periodontitis on erectile function and its possible mechanism. J Sex Med. 2011;8:25982605.

47. Sharma A, Pradeep AR, Raju PA. Association between chronic periodontitis and vasculogenic erectile dysfunction. J Periodontol. 2011;82:1665-1669.

How to cite this article: Mesa F, Arrabal-Polo MA, Magan-Fernández A, et al. Patients with periodontitis and erectile dysfunction suffer a greater incidence of major adverse cardiovascular events: A prospective study in a Spanish population. J Periodontol. 2022;1-10. https://doi.org/10.1002/JPER.21-0477 